\title{
PREECLAMPSIA SEVERA: RESTRICCIÓN DEL CRECIMIENTO INTRAUTERINO Y DESENLACES PERINATALES EN GESTACIONES PRETÉRMINO
}

Javier S. Llanos Buelvas MD*, José L. Rojas Arias MD**, Edgar Acuña Osorio MD***, Saulo Molina Giraldo MD****

\section{Resumen}

Objetivo: describir la frecuencia de complicaciones perinatales en gestaciones pretérmino de madres con preeclampsia severa con y sin restricción del crecimiento intrauterino (RCIU). Materiales y métodos: estudio descriptivo en embarazo único, preeclampsia severa y parto entre 24 y 34 semanas, con y sin RCIU, entre enero 2007 y diciembre 2009. Se realizó monitoría fetal cada 48 horas, perfil biofísico fetal (PBF) dos veces por semana y Doppler fetoplacentario semanal. Resultados: se estudiaron 55 pacientes con edad promedio de 29.6 (DE 6.4) años, media de edad gestacional de 29.9 (DE 2.9) semanas, menor en el grupo con RCIU 28.8 (DE.3.1) vs 31.2 (DE 2.0). Hubo RCIU en 30 (54,5\%) gestaciones, la mortalidad perinatal fue de 17 casos (31\%), 15 en embarazos con RCIU. La mayoría ocurrió en gestaciones por debajo de 28.6 semanas. Conclusiones: la mortalidad perinatal en preeclampsia en edad gestacional temprana es alta en nuestro hospital, en especial en presencia de RCIU. Es probable que esta asociación actúe en forma sinérgica sobre las complicaciones por prematurez. Al mejorar la atención pre y neonatal esperamos superiores resultados perinatales.

Palabras clave: mortalidad perinatal, preeclampsia, toxemia, restricción del crecimiento intrauterino, recién nacido pretérmino.

Abreviaturas: RCIU, retardo del crecimiento intrauterino; UCIN, unidad de cuidado intensivo neonatal; PRE-E, preeclampsia.

\section{SEVERE PRE ECLAMPSIA: INTRAUTERINE GROWTH RESTRICTION AND PERINATAL OUTCOME IN PRETERM GESTATIONS}

\begin{abstract}
Objective: to describe frequency of perinatal complications in preterm gestations associated to severe preeclampsia, with or without intrauterine growth restriction (IUGR). Materials and methods: this is a descriptive study of single pregnancies, severe preeclampsia and preterm birth between weeks 24 and 34, with and without IUGR, conducted
\end{abstract}

Fecha recibido: agosto 9 de 2010 - Fecha aceptado: septiembre 13 de 2010

* Residente II de Medicina Materno Fetal, Fundación Universitaria de Ciencias de la Salud. Hospital de San José Bogotá DC. Colombia.

** Especialista en Medicina Maternofetal. Jefe de Obstetricia, Hospital de San José, Bogotá DC. Colombia. Instructor Asociado, Fundación Universitaria de Ciencias de la Salud. Bogotá DC. Colombia.
*** Jefe del Servicio de Ginecoobstetricia, Hospital de San José. Profesor Asistente, Fundación Universitaria de Ciencias de la Salud. Bogotá DC. Colombia.

**** Especialista en Medicina Maternofetal, Hospital de San José y Coordinador del Diplomado en Ecografía Obstétrica. Instructor Asociado, Fundación Universitaria de Ciencias de la Salud. Bogotá DC. Colombia. 


\begin{abstract}
between January 2007 and December 2009. Fetal monitoring was conducted every 48 hours, fetal biophysical profile testing (BPP) twice a week and fetal and placental Doppler every week. Results: 55 patients with mean age 29.6 (SD 6.4) years were assessed; mean gestational age was 29.9 (SD 2.9) weeks, lower in the group with IUGR [28.8 (SD.3.1) vs 31.2 (SD 2.0)]. IUGR was present in 30 (54.5\%) pregnancies. Perinatal death occurred in 17 cases (31\%) 15 with IUGR. Most perinatal deaths occurred in pregnancies of less than $\mathbf{2 8 . 6}$ weeks. Conclusions: perinatal mortality rate in preeclampsia in early gestational age is high in our hospital, especially associated with IUGR. This association may act in a synergic manner on complications caused by prematurity. We expect better perinatal results as we improve prenatal and neonatal care delivery.
\end{abstract}

Key Hords: perinatal mortality rate, preeclampsia, toxemia, intrauterine growth restriction, preterm infant.

\section{Introducción}

La preeclampsia es una patología clasificada como un trastorno hipertensivo del embarazo con una frecuencia relativa alta en nuestro medio, siendo su incidencia de 5 al $10 \%$ de la población obstétrica general. ${ }^{1}$ Su curso clínico muchas veces presenta deterioro materno, fetal y neonatal. La PRE-E de inicio temprano representa un subgrupo de pacientes que tiene una frecuencia entre 5 y $20 \%$ de todos los casos. ${ }^{2}$

El parto y alumbramiento siguen siendo el único tratamiento efectivo y muchos casos de inicio temprano terminan en parto pretérmino, incrementando las complicaciones perinatales por prematuridad en los embarazos menores de 34 semanas. En estudios clínicos aleatorios los casos con PRE-E severa manejados de manera expectante lograron una prolongación significativa del embarazo, con una mejoría en el resultado perinatal y poco compromiso materno. ${ }^{3,4}$

Una de las complicaciones fetales que se relacionan con la PRE-E es la RCIU y algunas autoridades manifiestan que su presencia sumada a la PRE-E es una indicación para el parto después de un curso completo de corticoides para maduración pulmonar en embarazos pretérmino ${ }^{5,6}$; otros autores sugieren que la presencia de RCIU en embarazos lejos del término no es una indicación para el parto inmediato. ${ }^{2,7-9}$

El objetivo de este estudio es describir la frecuencia de complicaciones perinatales en gestaciones menores de 34 semanas de madres con PRE-E severa con y sin
RCIU, en el periodo 2007-2009 en el Hospital de San José de Bogotá DC, Colombia.

\section{Materiales y métodos}

Se realizó estudio descriptivo de una cohorte de pacientes gestantes con embarazo único que cumplieron los criterios para el diagnóstico de PRE-E severa, que además presentaron parto pretérmino entre 24 y 34 semanas, con o sin evidencia de RCIU, atendidas en el servicio de alto riesgo obstétrico del Hospital de San José durante el periodo $1^{\circ}$ de enero 2007 a 31 de diciembre 2009 , reclutando casos consecutivos. Se excluyen aquellos con malformación fetal mayor.

El cálculo de la edad gestacional se realizó en primera instancia por ecografias obstétricas tomadas en el primero o segundo trimestre y cuando no fue posible se estimó por fecha de última menstruación y ecografia de tercer trimestre.

El diagnóstico de PRE-E severa se definió por los criterios del Colegio Americano de Obstetricia y Ginecología basados en la presencia de PRE-E asociada con uno ó más de los siguientes: tensión arterial sistólica mayor de 160 ó diastólica mayor de $110 \mathrm{~mm} \mathrm{Hg}$, proteinuria mayor de cinco gramos en 24 horas, oliguria definida como menos de $500 \mathrm{ml}$ en 24 horas, alteraciones cerebrales o visuales (tinitus, fosfenos, cefalea), edema pulmonar, epigastralgia o dolor en cuadrante superior derecho del abdomen, alteraciones hepáticas de etiología no clara, trombocitopenia me- 
nor de 100.000 plaquetas y aumento de creatinina sérica $>1.2 \mathrm{mg} / \mathrm{dl} .^{5}$

Las pacientes con PRE-E severa recibieron manejo intrahospitalario, reposo e hidratación. Ante signos de inminencia de eclampsia como tinitus, fosfenos y epigastralgia se inició infusión de sulfato de magnesio a $1 \mathrm{~g}$ /hora. El tratamiento antihipertensivo se instauró ante cifra de tensión arterial mayor o igual a $160 / 110 \mathrm{~mm} \mathrm{Hg}$, con nifedipina $10 \mathrm{mg}$ oral cada seis horas y/o hidralazina $5 \mathrm{mg}$ intravenoso cada 20 minutos, máximo por cinco dosis hasta conseguir la meta de cifras tensionales. El control de paraclínicos diario incluyó cuadro hemático, recuento de plaquetas, creatinina sérica, parcial de orina y transaminasas hepáticas. Como pruebas de bienestar fetal, la monitoría se realizó cada 48 horas con perfil biofísico fetal (PBF) dos veces por semana y Doppler fetoplacentario semanal. El tratamiento para maduración pulmonar se realizó con betametasona intramuscular en dos dosis de $12 \mathrm{mg}$ cada una con intervalo de 24 horas según el protocolo del servicio.

La RCIU se definió ante un peso fetal estimado (PFE) por debajo del percentil 3, perímetro abdominal (PA) dos desviaciones estándar (DE) por debajo de la edad gestacional o PFE entre los percentiles décimo y tercero pero con alteración del Doppler fetoplacentario. Los estudios ultrasonográficos se tomaron con un equipo Xario prime ultrasound (Toshiba medical sistems corp. (C) en la unidad de medicina materno fetal del Hospital de San José por médicos ginecólogos con entrenamiento en ecografǐa obstétrica.

La medición del índice de líquido amniótico(ILA) resultó de la suma de los cuatro bolsillos mayores, uno en cada cuadrante abdominal. Se consideró normal si se encontraba entre los percentiles 5 y 95 de acuerdo con la edad gestacional y oligohidramnios o polihidramnios si estaba por debajo o por encima de los percentiles respectivos.

Para la medición del perfil biofísico fetal (PBF) se tomaron cinco parámetros: monitoría fetal sin estrés (NST), movimientos fetales, movimientos respiratorios, tono fetal e índice de líquido amniótico (ILA). A cada uno de ellos se les dio una puntuación de 2 que corresponde a una prueba normal, para lo cual deben cumplir con las siguientes características: 2 aceleraciones, 3 movimien- tos fetales en 30 minutos, por lo menos un movimiento respiratorio de 30 segundos en 30 minutos del examen, un movimiento de extensión-flexión de extremidad y un ILA mayor o igual a $5 \mathrm{~cm}$. Si no se cumplían los requerimientos anteriores se daba una puntuación de 0 . El PBF correspondió a la suma de cada uno de los parámetros.

Las mediciones de los flujos sanguíneos fetales por técnica Doppler se clasificaron según los protocolos de Vall Hebron $^{10}$, de la siguiente manera, teniendo en cuenta la edad gestacional:

- Tipo I: normal.

- Tipo II: aumento moderado de la resistencia placentaria sin redistribución y arteria umbilical con índice de pulsatilidad (IP) aumentado.

- Tipo III: aumento severo de resistencia placentaria sin redistribución arteria umbilical con flujo diastólico ausente.

- Tipo IV: signos de redistribución hemodinámica, arteria cerebral media con IP disminuido, (vasodilatación cerebral).

- Tipo V: alteración hemodinámica grave, arteria umbilical con flujo revertido y/o vena umbilical pulsátil y/o ductus venoso con flujo ausente o revertido.

Se definió el manejo expectante cuando transcurrieron más de 48 horas entre el ingreso hospitalario al hacer el diagnóstico y el momento de desembarazar. El resultado perinatal se evaluó según el peso al nacer, APGAR a los cinco minutos, necesidad de UCIN, días de estancia en UCIN (menor de 24 horas se consideró como un día completo), dosis terapéuticas de surfactante y mortalidad intrahospitalaria.

Este protocolo fue formulado de acuerdo con la reglamentación ética vigente (declaración de Helsinki, resolución 8430 de 1993 del Ministerio de Salud de Colombia). De igual manera fue evaluado y aprobado por el comité de investigaciones y ética de la Fundación Universitaria de Ciencias de la Salud.

Análisis estadístico: los datos para variables cuantitativas se presentan como promedios y desviaciones estándar o medianas y rango intercuartil según corresponda. Los datos categóricos se resumen como frecuencias absolu- 
tas y porcentajes. Se utilizó el programa STATA versión 10® para el análisis de los resultados.

\section{Resultados}

Se presentan los resultados de los dos primeros años de reclutamiento entre el $1^{\circ}$ de enero de 2007 y el 31 de diciembre de 2008. En este período se estudiaron 55 pacientes con PRE-E severa que cumplían criterios de inclusión. Las características de la población se describen en la Tabla 1. El promedio de edad materna fue de 29,6 (DE 6.4) años sin diferencias entre los grupos con y sin RCIU. La media de edad gestacional fue 29.9 (DE 2.9) semanas siendo menor en el grupo con RCIU 28.8 (DE.3.1) semanas vs 31.2 (DE 2.0) semanas en los embarazos sin RCIU. La presencia de RCIU se documentó en 30 (54,5\%) gestaciones (Figura 1).

Se realizó manejo expectante en 27 casos (49\%) con tiempo mediano entre el diagnóstico de PRE-E y el momento del parto de dos días (RIQ 1 a 6). La valoración del líquido amniótico previa a desembarazar fue anormal en once casos con RCIU $(36,7 \%)$ y cuatro $(16,6 \%)$ sin retardo.

El PBF con puntaje 10 se documentó en 36 valoraciones $(65,4 \%)$ al momento del diagnóstico y en $31(56,4 \%)$ en la prueba previa a desembarazar; esto en contraposición al puntaje 0 en 2 casos (3,6\%) al momento del diagnósti- co y $3(5,4 \%)$ antes del parto. La velocimetría Doppler al ingreso para los tipos I, II, IV y V se presentó en el $60 \%, 22 \%, 14 \%$ y $4 \%$ respectivamente. No se presentaron pacientes con Doppler tipo III. Los tipo V correspondían a dos casos con RCIU. Se documentaron cambios progresivos desde el ingreso hasta el momento de desembarazar sólo en el grupo con RCIU mientras que sin RCIU se presentaron dos casos con alteraciones en la velocimetría del flujo Doppler (tipos II y IV).

Los desenlaces neonatales se presentan en la Tabla 2. La mortalidad global fue de 17 casos (31\%), siendo más alta en el grupo de pacientes con RCIU (50\%) comparado con el grupo sin RCIU (8\%). Entre los primeros hubo mayores ingresos a UCIN, requerimiento de surfactante y APGAR bajo $(70,0 \%, 56,7 \%$ y $26,7 \%$ ) en comparación con aquellos sin RCIU ( $56 \%$, $28 \%$ y $4 \%)$.

La mortalidad se documentó con mayor frecuencia en edades gestacionales más tempranas, peso al nacer más bajo (en los fallecidos mediana $650 \mathrm{~g} v s 1580 \mathrm{~g}$ en los que sobrevivieron), APGAR bajo, presencia de oligohidramnios y Doppler IV y V. No sobrevivió ningún neonato con edad gestacional por debajo de 26 semanas (Figuras 2 y 3), ni los que tuvieron Doppler V (Tabla 3). No se presentaron casos de óbito fetal. Aún cuando no se planteó como objetivo de este estudio evaluar los desenlaces maternos, no reportamos casos de mortalidad.

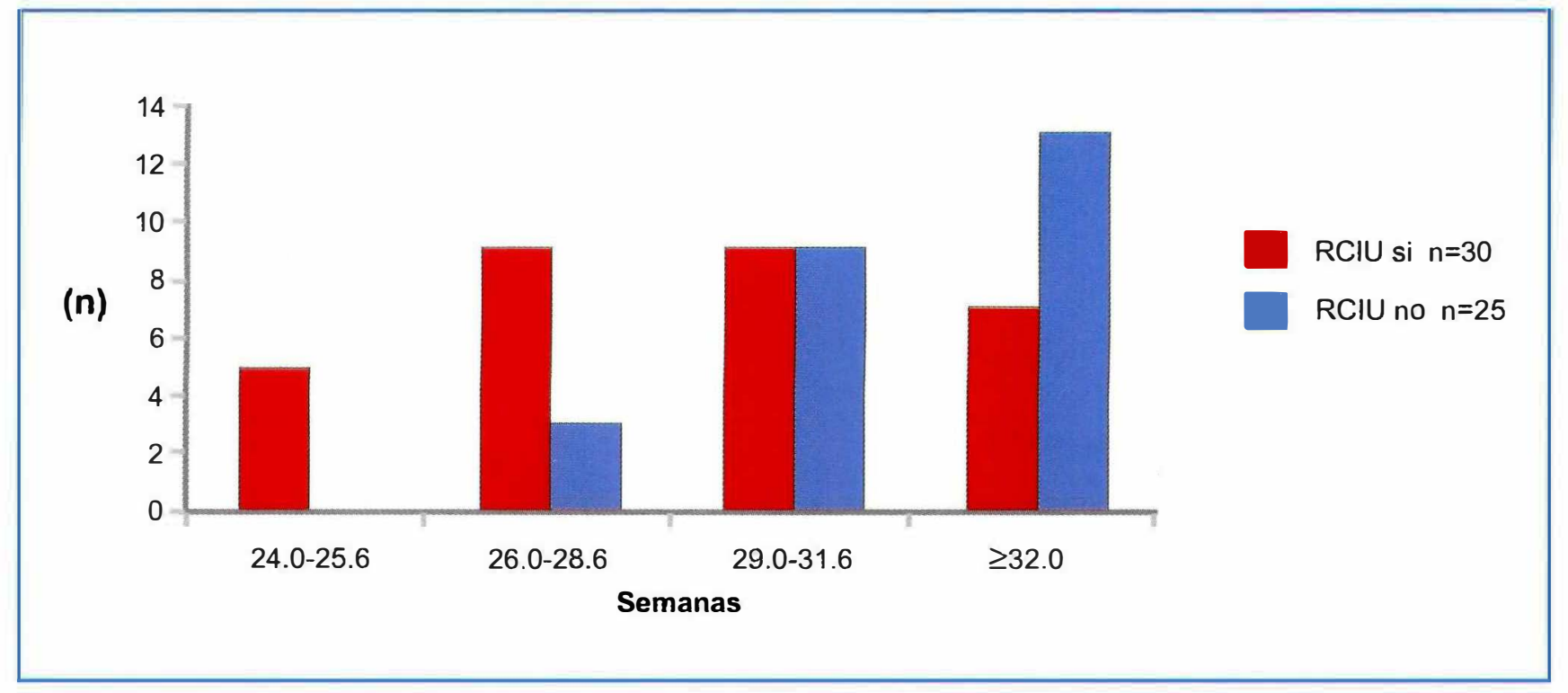

Figura I. RCIU según edad gestacional. 


\section{Tabla I. Características de la población}

\begin{tabular}{|c|c|c|c|c|c|c|}
\hline \multirow{2}{*}{$\begin{array}{l}\text { Caracteristicas } \\
\text { Edad (promedio, DE) } \\
\text { Rango edad }\end{array}$} & \multicolumn{2}{|c|}{$\underset{n=30}{\operatorname{Con} R C I U}$} & \multicolumn{2}{|c|}{$\begin{array}{c}\operatorname{Sin} R C I U \\
n=25\end{array}$} & \multicolumn{2}{|c|}{$\begin{array}{l}\text { Total } \\
n=55\end{array}$} \\
\hline & $\begin{aligned} 29.6 \\
17-\end{aligned}$ & $\begin{array}{l}(6.3) \\
40\end{array}$ & $\begin{array}{l}29.8 \\
15\end{array}$ & $\begin{array}{l}(6.6) \\
39\end{array}$ & $\begin{array}{l}29.6 \\
15-\end{array}$ & $\begin{array}{l}(6.4) \\
40\end{array}$ \\
\hline $\begin{array}{l}\text { Edad gestacional en semanas (promedio, DE) } \\
240 / 7-256 / 7 \\
260 / 7-286 / 7 \\
290 / 7-316 / 7 \\
\geq 320 / 7\end{array}$ & $\begin{array}{r}28.8 \\
5 \\
9 \\
9 \\
7\end{array}$ & $\begin{array}{l}(3.1) \\
(16.7) \\
(30) \\
(30) \\
(23.3)\end{array}$ & $\begin{array}{r}31.3 \\
0 \\
3 \\
9 \\
13\end{array}$ & $\begin{array}{l}(2) \\
(0) \\
(12) \\
(36) \\
(52)\end{array}$ & $\begin{array}{r}29.9 \\
5 \\
12 \\
18 \\
20\end{array}$ & $\begin{array}{l}(2.9) \\
(9.1) \\
(21.8) \\
(32.7) \\
(36.3)\end{array}$ \\
\hline Nuliparidad n (\%) & 13 & $(43.3)$ & 14 & $(56)$ & 27 & $(49)$ \\
\hline Primipaternidad n (\%) & 14 & $(46.6)$ & 12 & $(48)$ & 26 & $(47.2)$ \\
\hline $\begin{array}{l}\text { Tiempo entre el diagnóstico de preeclampsia } \\
\text { y el parto (mediana, RIQ*) en días }\end{array}$ & 2.5 & $(1-8)$ & 2 & $(2-5)$ & 2 & $(1-6)$ \\
\hline $\begin{array}{l}\text { Indicación para desembarazar } \\
\text { maternas } n(\%) \\
\text { fetales } \mathrm{n}(\%)\end{array}$ & $\begin{array}{r}21 \\
9\end{array}$ & $\begin{array}{l}(70) \\
(30)\end{array}$ & $\begin{array}{r}25 \\
0\end{array}$ & $\begin{array}{l}(100) \\
(0)\end{array}$ & $\begin{array}{r}46 \\
9\end{array}$ & $\begin{array}{l}(83.6) \\
(16.4)\end{array}$ \\
\hline $\begin{array}{l}\text { Liquido amniótico al ingresof } \\
\text { normal } n(\%) \\
\text { oligohidramnios } n(\%) \\
\text { polihidramnios } n(\%)\end{array}$ & $\begin{array}{r}24 \\
5 \\
1\end{array}$ & $\begin{array}{l}(80) \\
(16.6) \\
(3.4)\end{array}$ & $\begin{array}{r}21 \\
3 \\
0\end{array}$ & $\begin{array}{l}(87.5) \\
(12.5) \\
(0)\end{array}$ & $\begin{array}{r}45 \\
8 \\
1 \\
\end{array}$ & $\begin{array}{l}(83.3) \\
(14.8) \\
(1.9)\end{array}$ \\
\hline $\begin{array}{l}\text { Líquido amniótico al momento de desembarazar ๆ } \\
\text { normal } n(\%) \\
\text { oligohidramnios } n(\%) \\
\text { polihidramnios } n(\%)\end{array}$ & $\begin{array}{r}18 \\
11 \\
1\end{array}$ & $\begin{array}{l}(60) \\
(36.7) \\
(3.3)\end{array}$ & $\begin{array}{r}20 \\
4 \\
0\end{array}$ & $\begin{array}{l}(83.4) \\
(16.6) \\
(0)\end{array}$ & $\begin{array}{r}38 \\
15 \\
1\end{array}$ & $\begin{array}{l}(70.3) \\
(27.8) \\
(1.9)\end{array}$ \\
\hline $\begin{array}{l}\text { Perfil biofisico al momento de desembarazar } \S \mathbf{n}(\%) \\
0 \\
2 \\
4 \\
6 \\
8 \\
10 \\
\text { No aplica }\end{array}$ & $\begin{array}{r}2 \\
0 \\
0 \\
2 \\
4 \\
13 \\
9\end{array}$ & $\begin{array}{l}(6.7) \\
(0) \\
(0) \\
(6.7) \\
(13.3) \\
(43.3) \\
(30)\end{array}$ & $\begin{array}{r}1 \\
0 \\
0 \\
1 \\
3 \\
18 \\
2\end{array}$ & $\begin{array}{l}(4) \\
(0) \\
(0) \\
(4) \\
(12) \\
(72) \\
(8)\end{array}$ & $\begin{array}{r}3 \\
0 \\
0 \\
3 \\
7 \\
31 \\
11\end{array}$ & $\begin{array}{l}(5.4) \\
(0) \\
(0) \\
(5.4) \\
(12.7) \\
(56.4) \\
(20)\end{array}$ \\
\hline $\begin{array}{l}\text { Doppler al ingresott } \\
\text { tipo I } \\
\text { tipo II } \\
\text { tipo III } \\
\text { tipo IV } \\
\text { tipo V }\end{array}$ & $\begin{array}{r}10 \\
10 \\
0 \\
6 \\
2\end{array}$ & $\begin{array}{l}(35.7) \\
(35.7) \\
(0) \\
(21.4) \\
(7.2)\end{array}$ & $\begin{array}{r}20 \\
1 \\
0 \\
1 \\
0\end{array}$ & $\begin{array}{l}(91) \\
(4.5) \\
(0) \\
(4.5) \\
(0)\end{array}$ & $\begin{array}{r}30 \\
11 \\
0 \\
7 \\
2\end{array}$ & $\begin{array}{l}(60) \\
(22) \\
(0) \\
(14) \\
(4)\end{array}$ \\
\hline $\begin{array}{l}\text { Doppler al momento de desembarazartt } \\
\text { tipo I } \\
\text { tipo II } \\
\text { tipo III } \\
\text { tipo IV } \\
\text { tipo V }\end{array}$ & $\begin{array}{l}7 \\
9 \\
0 \\
9 \\
3\end{array}$ & $\begin{array}{l}(25) \\
(32.1) \\
(0) \\
(32.1) \\
(10.7)\end{array}$ & $\begin{array}{r}20 \\
1 \\
0 \\
1 \\
0\end{array}$ & $\begin{array}{l}(91) \\
(4.5) \\
(0) \\
(4.5) \\
(0)\end{array}$ & $\begin{array}{r}27 \\
10 \\
0 \\
10 \\
3\end{array}$ & $\begin{array}{l}(54) \\
(20) \\
(0) \\
(20) \\
(6)\end{array}$ \\
\hline
\end{tabular}

* RIQ: rango intercuartil. † Sobre 54 datos. $¥$ Sobre 54 datos. $§$ Perfil biofísico se realiza a partir de las 28 semanas. †† Sobre 50 datos $(28$ Doppler realizados en pacientes con RCIU y 22 en pacientes sin RCIU). II Manejo expectante: > 48 horas desde el diagnóstico hasta desembarazar. 


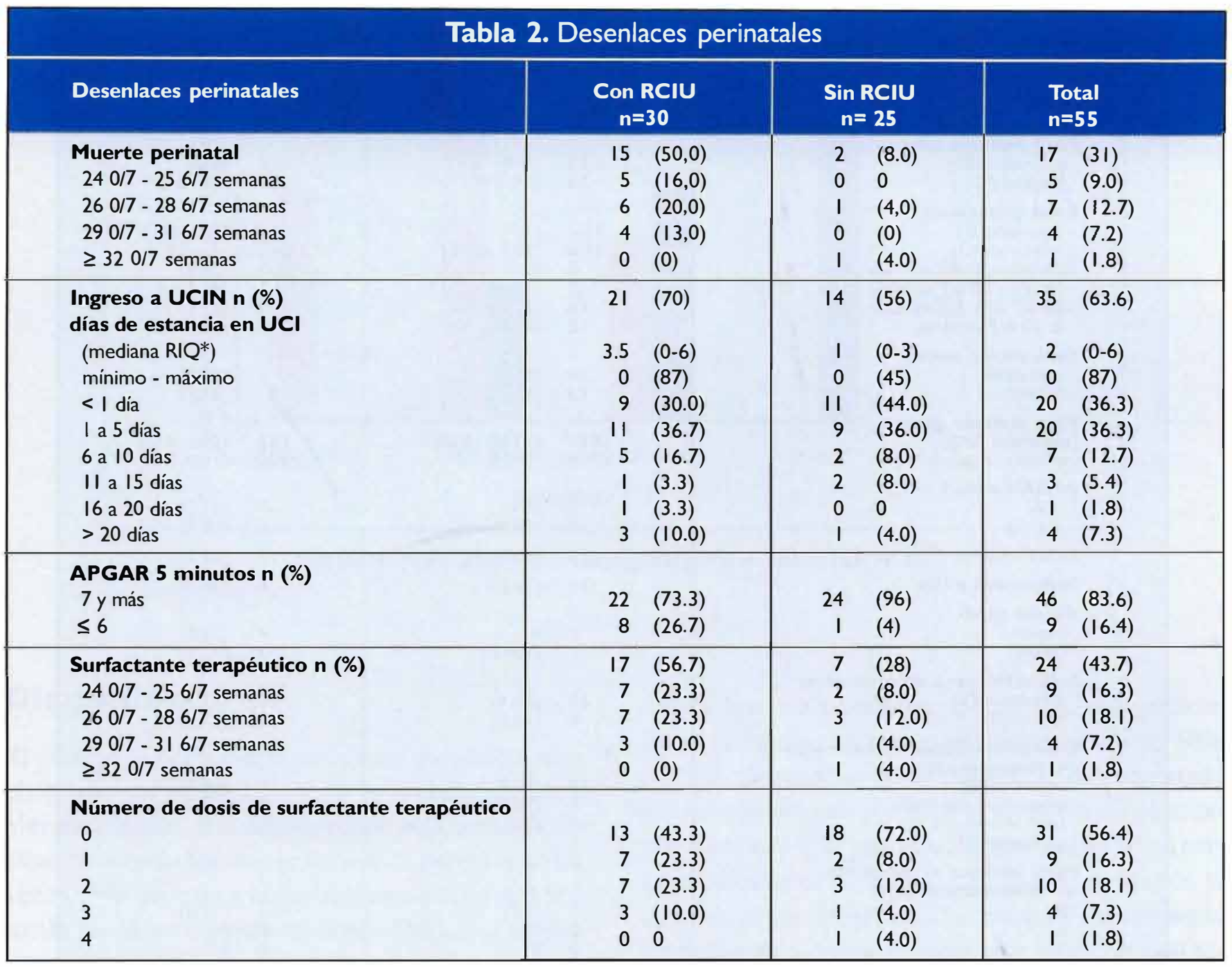

* RIQ: rango intercuartilico.

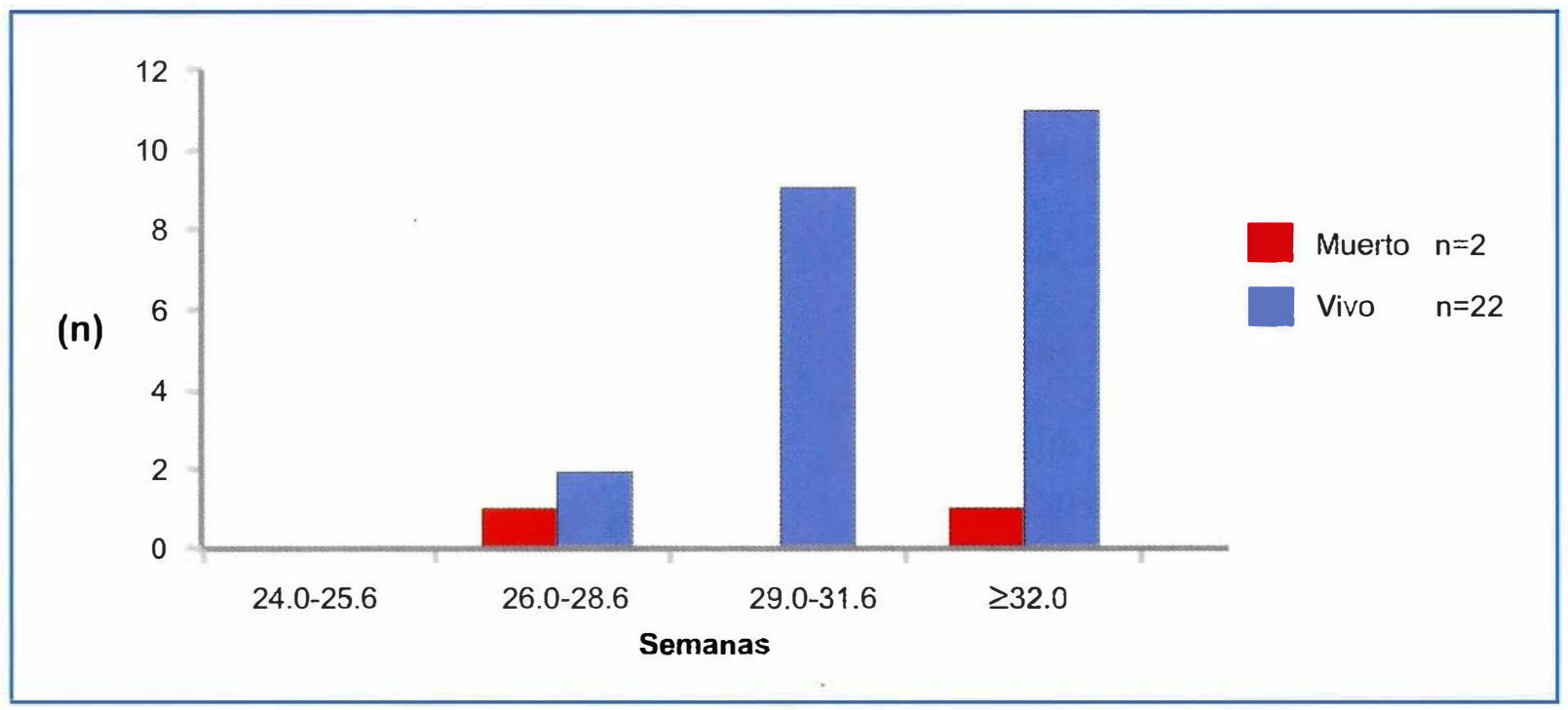

Figura 2. Mortalidad según edad gestacional en casos sin RCIU. 


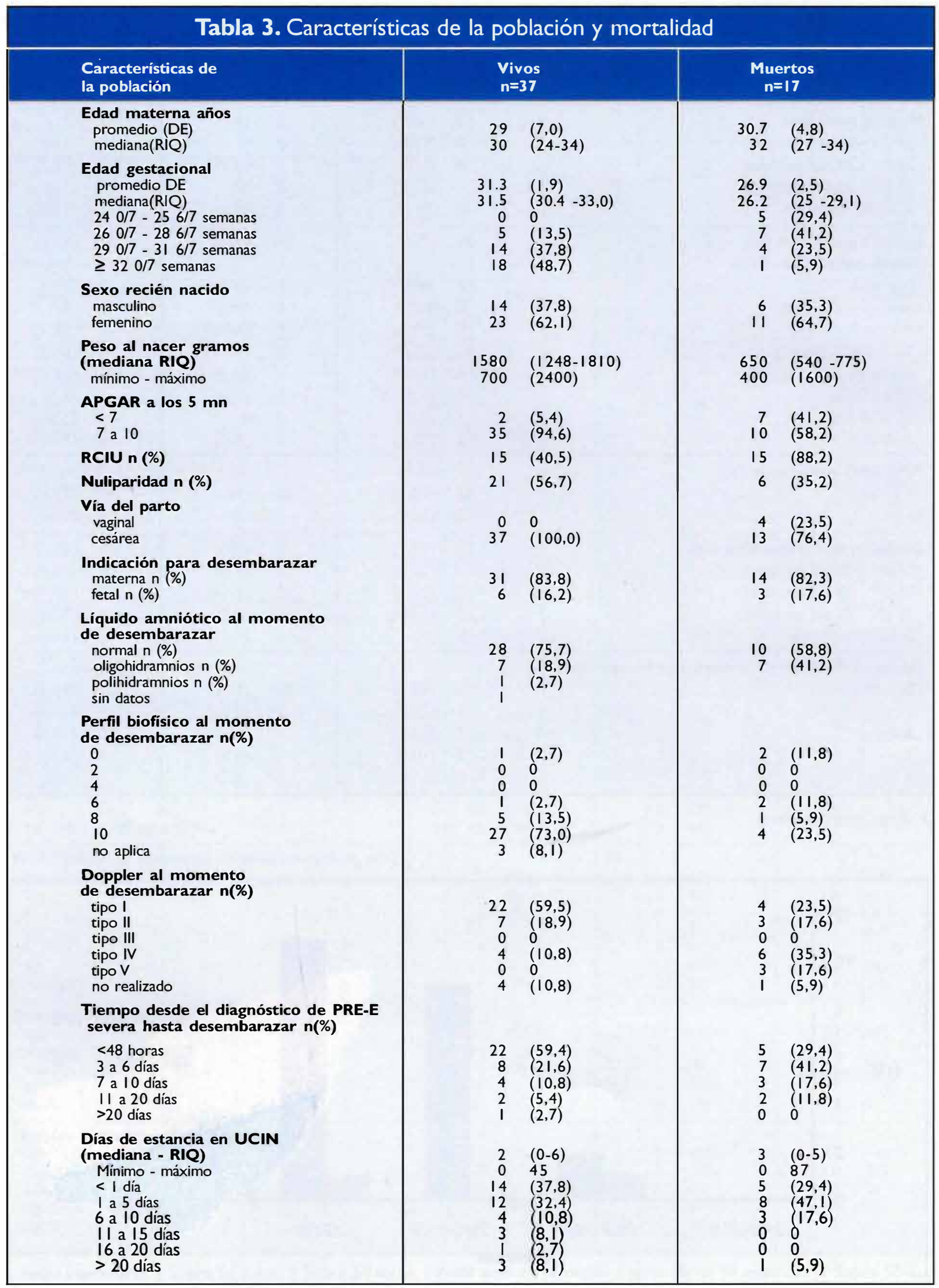

UCIN: Unidad de cuidados intensivos neonatales. 


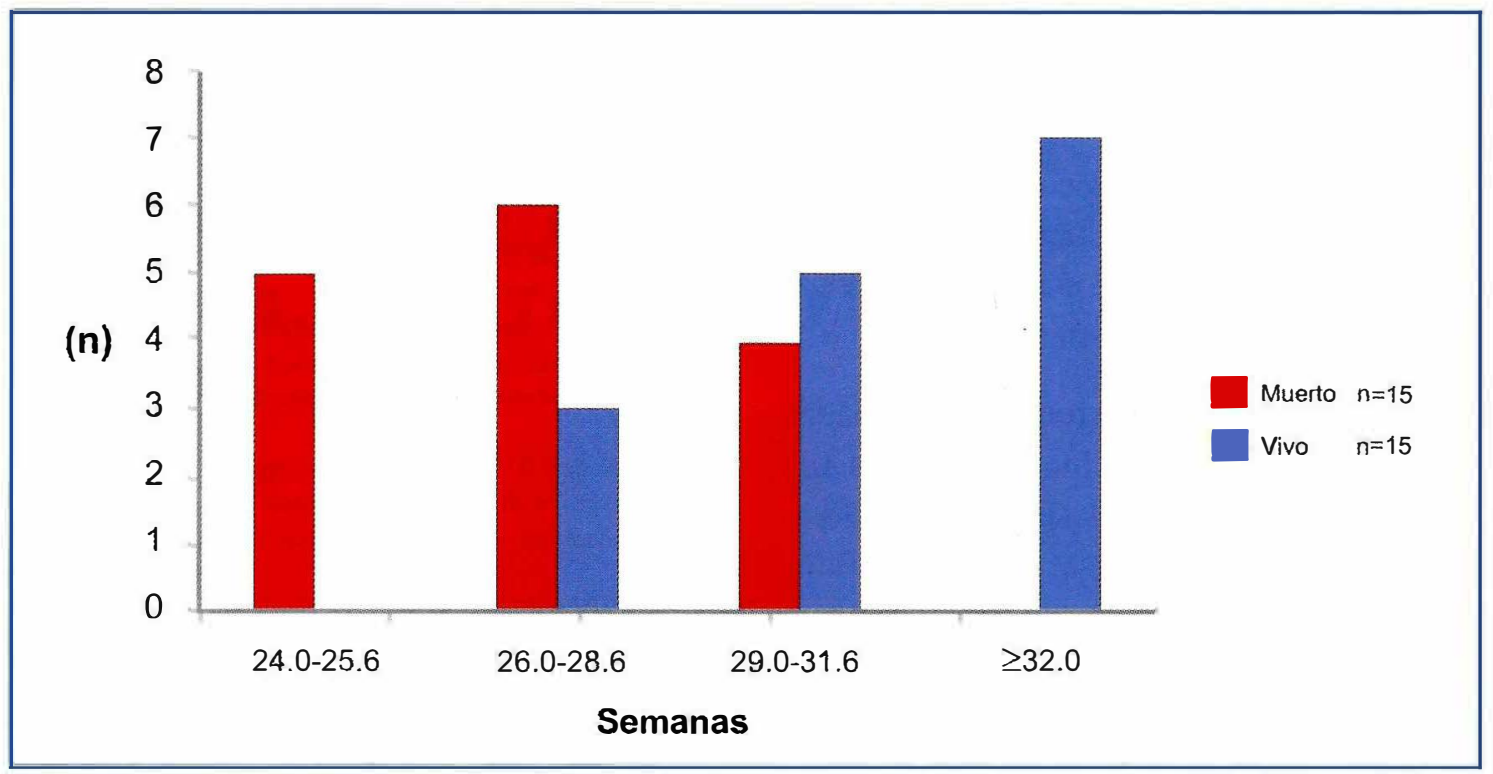

Figura 3. Mortalidad según edad gestacional en casos con RCIU.

\section{Discusión}

El manejo de la PRE-E sigue siendo un desafío para el obstetra por las implicaciones en la madre, el feto y el recién nacido; el resultado puede cubrir un amplio espectro siendo bueno por fortuna la mayoría de las veces, pero malo para la madre y en especial el feto y recién nacido en algunas ocasiones. Debido al amplio rango de resultados maternos y perinatales, algunos autores la han clasificado en PRE-E de inicio temprano (antes de 34 semanas) y tardío (después de 34) con diferentes etiologías, expresión clínica y resultado materno-fetal. ${ }^{11}$ La de inicio temprano se caracteriza muchas veces por presentar manifestaciones clínicas más severas, cambios en el flujo sanguíneo de las arterias uterinas (con muesca y aumento de los índices de pulsatilidad a la velocimetría Doppler), elevación de resistencia de los vasos placentarios (aumento de relación sístole/diástole, ausencia o aun inversión del flujo al final de la diástole de arterias umbilicales) y RCIU. ${ }^{9}$ En este escenario la mortalidad perinatal ha sido reportada en un 5,4\% por Haddad y colaboradores quienes estudiaron pacientes con PREE severa y RCIU severo y no severo ${ }^{12}$; sin embargo, estos datos contrastan con los hallazgos de nuestro estudio en el que tuvimos una mortalidad global del $31 \%$ (17/54) incluyendo casos con y sin RCIU. Ana- lizando la mortalidad del grupo con RCIU encontramos que es aún mayor en nuestra población siendo del $50 \%$ (15/30). Cifras altas de mortalidad se han reportado pero en poblaciones con PRE-severa y prematurez extrema entre 21 y 27 semanas, llegando al $43 \%{ }^{13}$; a partir de estos datos exploramos en nuestra población la mortalidad por debajo de 27 semanas y encontramos diez muertes perinatales entre once casos, lo cual representa una mortalidad del $90 \%$ en este grupo frente a una mortalidad del 16\% (7/43) en las gestaciones mayores o iguales a 27 semanas. Shear y colaborado$\mathrm{res}^{8}$ reportaron un punto de corte de 30 semanas en el cual el manejo expectante de preeclámpticas con RCIU presentaba beneficios sobre el manejo no conservador. Nosotros encontramos que en las pacientes con PREE severa y RCIU no se presentaron casos de mortalidad perinatal a partir de las 32 semanas de embarazo. Sabemos que los índices de mortalidad perinatal se relacionan con prematurez, complicaciones asociadas y experiencia de su manejo en la UCIN, y en edades gestacionales tempranas es alta en nuestro centro de atención, en especial en presencia de RCIU. Es probable que esta asociación actúe en forma sinérgica sobre las complicaciones por prematurez y en la medida que mejore la atención pre y neonatal también esperaríamos superiores resultados perinatales. 
Los datos parciales de nuestro trabajo muestran que en los casos con RCIU y edades gestacionales tempranas hubo mayores tasas de mortalidad, ingresos a UCIN y necesidady dosis de surfactante terapéutico, comparados con los casos equiparables en edades gestacionales sin RCIU. Estos sólo se observaron hasta una edad gestacional de 32 semanas, a partir de la cual disminuyen.

En nuestro estudio no se consideraron comorbilidades maternas y complicaciones como síndrome de HELLP, CID, edema pulmonaro abrupcio placentario que pudieran contribuir a explicar los desenlaces neonatales. Tampoco hacemos mención de los casos que no completaron esquema de maduración pulmonar fetal.

Estos resultados no son extrapolables a otras unidades de alto riesgo obstétrico; describen de manera exploratoria los resultados basados en registros hospitalarios del periodo 2007-2008 sin hacer análisis por intervalos de tiempo, pudiendo variar la incidencia de complicaciones perinatales entre un año y otro. De otra parte, el Hospital de San José es centro de referencia de pacientes con patologías de gran complejidad, que puede haber incidido en la mayor frecuencia de desenlaces adversos observados de los recién nacidos cuando nos comparamos con otras series.

Faltarían más estudios clínicos con diseños aleatorios controlados para evaluar el impacto de la PRE-E severa y RCIU en la mortalidad y otros desenlaces perinatales, teniendo en cuenta si se ofrece manejo expectante o convencional, como lo proponen algunas publicaciones. ${ }^{3,46} \mathrm{La}$ mortalidad perinatal en PRE-E en edades gestacionales tempranas es alta en nuestro centro de atención, sobre todo en presencia de RCIU. La disponibilidad de mejores métodos y equipos en vigilancia fetal y cuidados intensivos neonatales podría contribuir a obtener mejores resultados perinatales, no sólo en casos de prematuridad extrema sino en aquellos asociados con RCIU.

\section{Referencias}

1. Cifiuentes R. Hipertensión arterial y embarazo. En: Obstetricia de alto riesgo. $4^{\circ}$ ed. Colombia: Aspromédica; 1994. p. 525-610.

2. Huppertz B. Placental origins of preeclampsia challenging the current hypothesis. Hypertension. 2008; 51(4): 970-5.

3. Sibai BM, Mercer BM, SchiffE, Friedman SA. Aggressive versus expectant management of severe preeclampsia at 28 to 32 weeks' gestation: a randomized controlled trial. Am J Obstet Gynecol. 1994; 171(3):818-22.

4. Odendaal HJ, Pattinson RC, et al. Aggressive or expectant management of patients with severe preeclampsia between 28-34 weeks' gestation: a randomized controlled trial. Obstet Gynecol. 1990; 76(6): 1070-4.

5. ACOG technical bulletin. Hypertension in pregnancy. Number 219 January. Committee on Technical Bulletins of theAmerican College of Obstetricians and Gynecologists. Int J Gynaecol Obstet. 1996 May;53(2): 175-83

6. Chammas MF, Nguyen TM, Li MA, Nuwayhid BS, Castro LC. Expectant management of severe preterm preeclampsia: is intrauterine growth restriction an indication for immediate delivery?. Am J Obstet Gynecol. 2000; 183(4):853-8.

7. Schiff E, Friedman SA, Sibai BM. Conservative management of severe preeclampsia remote from term. Obstet Gynecol. 1994; 84(4): 626-30.

8. Shear RM, Rinfret D, Leduc L. Should we offer expectant management in cases of severe pretern preeclampsia with fetal growth restriction?. Am J Obstet Gynecol. 2005;192(4):1119-25.

9. Ganzevoort W, Rep A, Bonsel GJ, Fetter WPF, van Sonderen L, De Vries JI, et al. A randomised controlled trial comparing two temporising management strategies, one with and one without plasma volume expansion, for severe and early onset pre-eclampsia. BJOG. 2005; 112(10): 1358-68.

10. Figueras F, Gratacós E. Alteraciones del crecimiento fetal. En: Medicina fetal Buenos Aires: Editorial médica panamericana; 2007.

11. Von Dadelszen P, Magee LA, Roberts JM. Subclassification of preeclampsia Hypertens Pregnancy. 2003;22(2):143-148.

12. Haddad B, Kayem G, Deis S, Sibai BM. Are perinatal and maternal outcomes different during expectant management of severe preeclampsia in the presence of intrauterine growth restriction?. Am J Obstet Gynecol. 2007; 196(3):237 e1-237.e5.

13. Bombrys AE, Barton JR, Nowacki EA, et al. Expectant management of severe preeclampsia at less than 27 weeks' gestation: maternal and perinatal outcomes according to gestational age by weeks at onset of expectant management. Am J Obstet Gynecol. 2008; 199(3): 247.e1247.e6 